\title{
The Effects of Nitrogen and Oxygen Atmosphere on the Photoconductivity of Trimethyl Phenyl Diamine Thin Films
}

\author{
Nazrul Anuar Nayan ${ }^{1}$, Khairul Anuar A. Rahman ${ }^{2}$ \\ ${ }^{1}$ Center for Integrated Systems Engineering and Advanced Technologies, Faculty of Engineering and Built Environment, \\ Universiti Kebangsaan Malaysia, UKM Bangi Selangor, Malaysia \\ ${ }^{2}$ Department of Electronics and Computer Technology Faculty of Engineering Technology \\ Universiti Teknikal Malaysia Melaka, Malaysia
}

\begin{tabular}{l}
\hline \hline Article Info \\
\hline Article history: \\
Received Feb 23, 2018 \\
Revised May 05, 2018 \\
Accepted May 20, 2018 \\
\hline
\end{tabular}

Keywords:

Electron mobility Organic semiconductor

Photoconductivity

Residual decay

TPD

\begin{abstract}
Organic materials were previously used as insulators in electrical technology. These materials, however, are currently used as conductors once their photoconductivity is confirmed and studied. From the literature, it has shown that the photoconductivity of trimethyl phenyl diamine (TPD) increases in the air and decreased in the atmosphere of the vacuum. To the best of our knowledge, there is no detailed study of the effects of gas in the air that affect TPD photoconductivity. In this study we investigate the effects of nitrogen $\left(\mathrm{N}_{2}\right)$ and oxygen $\left(\mathrm{O}_{2}\right)$ gases on photoconductivity, degradation and residual decay of photoconductivity for thin film TPD. The results of the study show that in the atmosphere of $\mathrm{O}_{2}$, TPD produces about seven times higher photoconductivity compared to $\mathrm{N}_{2}$ conditions. It also shows that, $\mathrm{N}_{2}$ and $\mathrm{O}_{2}$ provide more effective response time during photoconductivity residual decay. Photoconductivity degradation occurs in all conditions and its recovery takes more than 65 hours.
\end{abstract}

Copyright $\odot 2018$ Institute of Advanced Engineering and Science. All rights reserved.

\section{Corresponding Author:}

Nazrul Anuar Nayan,

Center for Integrated Systems Engineering and Advanced Technologies,

Faculty of Engineering and Built Environment,

Universiti Kebangsaan Malaysia,

UKM Bangi Selangor, Malaysia

Email: nazrul@ukm.edu.my

\section{INTRODUCTION}

In 1954, Hiroo Inokuchi and Hideo Akamatsu conducted ground-breaking studies that systematically investigated the intermolecular electrical conductivity of violanthrone, an organic molecule that consists of nine benzene rings. Violanthrone acts like a semiconductor in a manner that is similar to that of inorganic materials [1]. This finding is a stepping stone for the development of organic semiconductor applications, such as displays, photovoltaics, and electronic components. Organic field-effect transistors and organic light-emitting diodes remain as the fundamental electronic building blocks of organic electronic circuits [2]. Compared with inorganic semiconductors, organic semiconductors are more easily tailored for specific applications [3]. For example, compounds that contain conjugate bonds, such as $-\mathrm{C}=\mathrm{C}-\mathrm{C}=$, have large optical nonlinearities and thus have potential applications in optoelectronics. In addition, organic semiconductors have low cost, mechanical flexibility, synthetic versatility, solution-processability, and highpurity; these materials can be tailored into high-performance photoconductive thin films [4], [5]. Molecular design for a variety of molecular structures is easily performed by combining atoms with different internal and intermolecular forces. Chemical formulas are changed to suit a specific application. The bandgaps of organic semiconductors are easier to change than those of inorganic semiconductors.

Trimethyl Phenyl Diamine (TPD) is widely used as a material for typical photoconductive organic films given its simple structure and fundamental photoconductivity [6]. The steady-state photoconductivity of 
other organic semiconductors has also been investigated [7]. TPD, as shown in Figure 1, has two sets of triphenyl amine with an ionization potential of approximately $5.4 \mathrm{eV}$. TPD also possesses an aromatic amine compound with $\sim 10^{-3} \mathrm{~cm}^{2} / \mathrm{Vs}$ positive hole mobility. Holes are the dominant carrier in TPD films. TPD is a representative charge transport material given its relatively high mobility, which can be increased by one digit via doping. The use of TPD as a hole transport layer in organic electroluminescence has been recently studied [8]. Furthermore, the hopping photoconductivity and reasonable physical parameters of TPD have been found to consist of hopping relaxation time, recombination time, and diffusion coefficient of charge carriers [9]. These characteristics contribute to the photoconductivity of TPD.

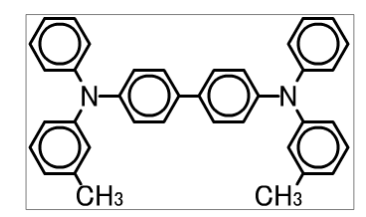

Figure 1. Chemical structure of TPD

Similar to a general semiconductor, the band theory can be applied when an electron or a positive hole becomes the carrier in an organic polymeric semiconductor. However, unlike in crystalline semiconductors, various localized levels can form in organic polymeric conductors because of its irregular structure. Therefore, organic conductors resemble an amorphous semiconductor with an extremely rugged conduction and valence band, as well as a band gap value that remains constant. From the previous study, it showed that the photoconductivity of organic semiconductor films tends to increase in air and decrease in a vacuum condition [9]. To clarify what are the gases involved, in this study, the effects of air, oxygen, and nitrogen on the photoconductivity of TPD film were investigated. Then the photoconductivity degradation which occurs during irradiation has also been examined. Finally, and residual decay after the interception has also been studied. Residual decay is the remaining photocurrent after the light is intercepted. By understanding the effect of $\mathrm{O}_{2}$, we have understood that the photoconductivity is TPD increases in the air atmosphere is due to the content of $\mathrm{O}_{2}$ in it. The results could be used for post-power shortage situation where immediate emergency light is needed and should be extra long-lasting.

\section{METHOD}

Prior to TPD film preparation, the glass substrate (Corning 7059) is cleaned using ultrasonic cleaning at 10,50 , and $100 \mathrm{kHz}$ in both acetone and ethanol at 20 cycles per solvent. Then, a vacuum evaporation system (Ulvac Corp. K.K EBH-6 type) as shown in Figure 2 is used to deposit a $0.5 \mu \mathrm{m}$ thick TPD thin film onto the glass substrates via the thermal evaporation technique.

Deposition is conducted in a vacuum condition of approximately $2 \times 10^{-6}$ Torr at room temperature. The Au electrode is deposited in a vacuum deposition (resistive heating) with a mask, and the result of the final deposition of sample and Au electrodes, which is a gap cell type is shown in Figure 3. Optical transmittance is measured by spectrophotometer (Shimadzu Corporation UV-3200S). The film thickness can be estimated from the refractive index of each minimum, maximum value of the sample.

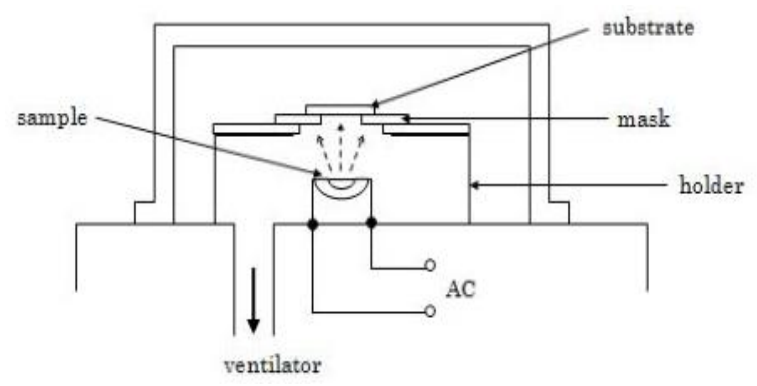

Figure 2. Vacuum evaporation diagram

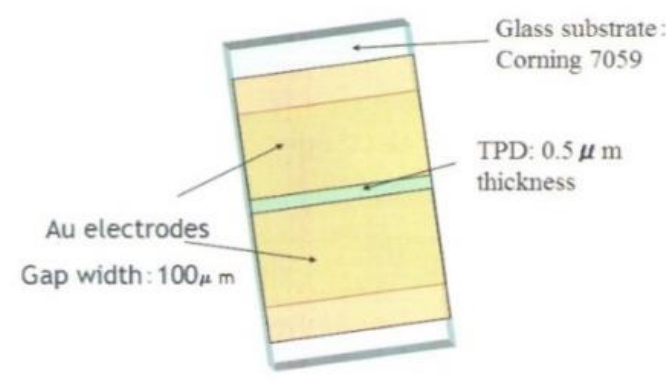

Figure 3. TPD thin film gap cell type 
Figure 4 shows the main equipment used in this study. The apparatus consists of a cryostat, $\mathrm{He}-\mathrm{Cd}$ laser, measurement display, and plotting software. The cryostat is an air-tight enclosure in which the samples are placed during measurement. First, Ag powder paste is used to mount the gold wire on the electrodes of the sample. The sample is then pasted on the wall of the cryostat. Next, the gold wire is connected with an external measuring terminal and wiring for the DC power source. After the sample is installed, the cryostat is vacuumed with the rotary pump. Neutral density filter sets are used to obtain the desired density. The laser enters through a point in the glass windows. A three-way valve is connected to the cryostat to switch between the rotary pump-cryostat and the gas canister-cryostat. $\mathrm{O}_{2}$ and $\mathrm{N}_{2}$ gases are then injected.

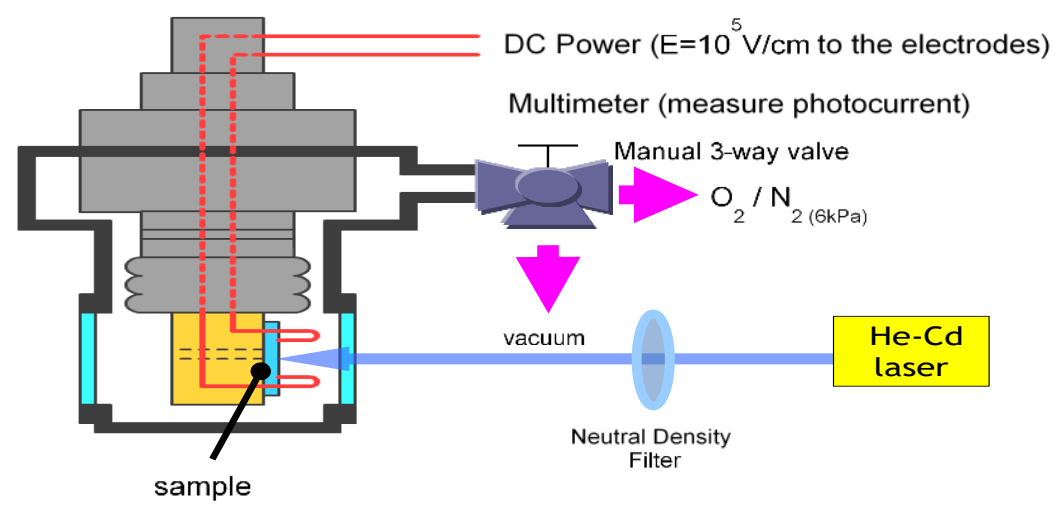

Figure 4. Diagram of measurement method

A temperature controller and frozen compressor are not used because the experiments were conducted at room temperature, $300 \mathrm{~K}$. The internal temperature of the cryostat is measured using a thermocouple. Labview is used for accurate and long-time data plotting. In this study, the majority of the data are obtained after $96 \mathrm{~h}$. The changes in the photocurrent are easier to observe when the laser is alternately turned off or on. The shortest measurement speed is set to $0.5 \mathrm{~s}$.

An electric field of $10^{5} \mathrm{~V} / \mathrm{cm}$ is applied to the electrodes of the sample. Photocarriers are induced by irradiation; with a single power of $72 \mathrm{~mW} / \mathrm{cm}^{2} \mathrm{He}-\mathrm{Cd}$ laser at $3.82 \mathrm{eV}(\lambda=325 \mathrm{~nm})$. The estimated charge carrier generation rate is $7.2 \times 10^{21} \mathrm{~cm}^{-3} \mathrm{~s}^{-1}$. The photocurrent in TPD, which is set in the cryostat, is measured in vacuum, air, $\mathrm{O}_{2}$, and $\mathrm{N}_{2}$ conditions. Experimental data are fitted to stretch exponential functions.

For the photon frequency, $v$ is calculated as $9.23 \times 10^{14} \mathrm{~s}^{-1}(h v=3.82 \mathrm{eV})$ at $1 \mathrm{~mW} / \mathrm{cm}^{2}$. The number of photons deduces from the laser power is divided by $h v$ and is

$$
N_{0}=0.001\left[\mathrm{~W} \mathrm{~cm}^{-2}\right] /\left(9.23 \times 10^{14}\left[\mathrm{~s}^{-1}\right] \cdot 6.63 \times 10^{-34}[\mathrm{Js}]\right)=1.63 \times 10^{15}\left[\mathrm{~cm}^{-2} \mathrm{~s}^{-1}\right] .
$$

Given that the reflection $\mathrm{R}$ is $30 \%$, the thickness of sample is $0.5 \mu \mathrm{m}$ and the absorption coefficient $\alpha=10^{5}$ $\mathrm{cm}^{-1}$, the photogeneration rate is calculated as follows:

$$
G=\mathrm{N}_{0}(1-R) \cdot \alpha=1.63 \times 10^{18} \cdot 0.7 \cdot 10^{5}=1.14 \times 10^{20}\left[\mathrm{~cm}^{-3} \mathrm{~s}^{-1}\right]
$$

The increase in the current after the laser irradiation $\left(f_{l}(t)\right)$ and the attenuation of the current after the laser is intercepted $\left(f_{2}(t)\right)$ and studied by fitting the graph with the stretch exponential functions, respectively.

$$
f_{l}(t)=A\left(1-\exp \left[-\left(t_{\mathrm{T}}\right)^{\beta}\right]\right)
$$

and

$$
\left.f_{2}(t)=A \exp \left[-(t /)_{\mathrm{T}}\right)^{\beta}\right]
$$

Here, ${ }_{\mathrm{T}}[\mathrm{sec}]$ is the effective response time to show the response speed and $\beta$ is the dispersion parameter. For example, in $f_{l}(t),{ }_{\mathrm{T}}=100 \mathrm{~s}$ and $\beta=0.26$, whereas in $f_{2}(t), \mathrm{T}_{\mathrm{T}}=1.24 \mathrm{~s}$ and $\beta=0.47$. In $f_{l}(t)$, the value of ${ }_{\mathrm{T}}$ is the time until saturation. This value depends on the laser power. A higher power indicates a smaller ${ }_{\mathrm{T}}$ value. The stretched exponential function is a common model for describing relaxation data. This model has been used extensively to represent the stress-relaxation of biological tissues and other systems [10]. 


\section{RESULTS AND ANALYSIS}

As shown in Figure 5, the photocurrent increases when air is injected into the cryostat in vacuum condition at $\mathrm{t}=30 \mathrm{~min}$. In air, the photocurrent in TPD is $5 \mathrm{nA}$ to $8 \mathrm{nA}$ compared with that in vacuum, which is approximately $2.5 \mathrm{nA}$. The photocurrent returns to its original value when the TPD is returned to the vacuum condition. The photocurrent increases again after the air is replaced by vacuum in the next cycle at $\mathrm{t}=300 \mathrm{~min}$. Given that this behavior is reversible, it is a physical rather than a chemical reaction.

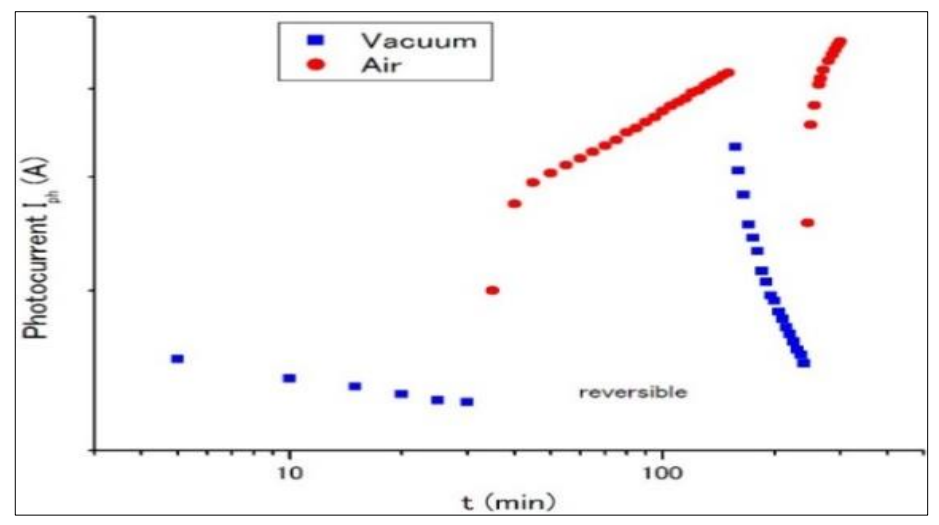

Figure 5. Photoconductivity in vacuum and air conditions during light irradiation

Air is composed of $78 \%$ molecular $\mathrm{N}_{2}, 20 \% \mathrm{O}_{2}, 1.9 \% \mathrm{Ar}$, and small amounts of $\mathrm{Ne}, \mathrm{He}, \mathrm{CH}_{4}, \mathrm{Kr}, \mathrm{H}$, $\mathrm{N}_{2} \mathrm{O}$, and Xe. Air contains variable concentrations of water vapor $(\mathrm{H} 2 \mathrm{O})$, ozone $\left(\mathrm{O}_{3}\right), \mathrm{CO}_{2}$, and $\mathrm{SO}_{2}$. $\mathrm{O}_{2}$ is the most active gas for chemical or physical reactions.

The photoconductivity of TPD in vacuum is measured before introducing air and gas. For this experiment, the photocurrent measurements are performed when the laser is turned on and off. The changes in the photocurrent of the samples are observed in the dark, after irradiation, and after laser interception. Table 1 shows the processing times when the laser is on and off. The first light is on after 5 min, and the final interception or light off is after $97 \mathrm{~h}$.

Figure 6 shows the photocurrent of TPD films in vacuum conditions. The dark current is approximately $25 \mathrm{pA}$. Irradiation begins at $300 \mathrm{~s}$. The photocurrent increases to a peak of $1.5 \mathrm{nA}$. Photoconductivity degradation occurs after the photocurrent reaches the maximum value. Residual decay is observed after the light is turned off. As a reference, the photocurrent in a vacuum as shown in Figure 6 is measured with the light on/off as mentioned in Table 1. The values are shown as irradiation (laser on) and interception (laser off). Since the amount of data is huge, it seems like the photocurrent has two data at the same time $(\mathrm{t})$. Figure 6 is to demonstrate the overview pattern for photocurrent during irradiation, degradation, interception and residual decay in vacuum atmosphere.

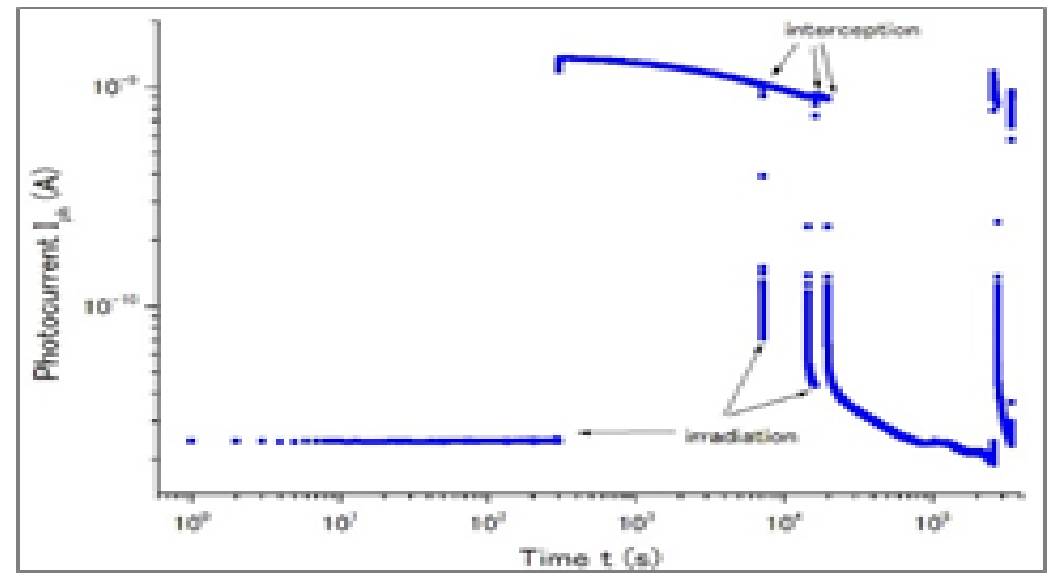

Table 1. Laser On/Off Time

\begin{tabular}{cc}
\hline \multicolumn{2}{c}{ Method } \\
\hline Time & Laser \\
[hh:mm:ss] & On/Off \\
00:05:00 & ON \\
02:00:00 & OFF \\
02:01:00 & ON \\
$04: 00: 00$ & OFF \\
$04: 30: 00$ & ON \\
$05: 30: 00$ & OFF \\
$72: 00: 00$ & ON \\
$77: 00: 00$ & OFF \\
96:00:00 & ON \\
97:00:00 & OFF \\
\hline
\end{tabular}

Figure 6. Photocurrent in vacuum conditions 
A separate experiment is conducted in air atmospheric condition. Then, the results for air and vacuum conditions are compared. Figure 7 shows the photocurrents under the air and vacuum conditions. No significant difference is observed before irradiation (dark). The maximum value of photocurrent in air is 1.5 times more than that in vacuum. After the first interception $(t=2 \mathrm{~h})$, the photocurrent increases in the air condition, which is different from that in the vacuum.

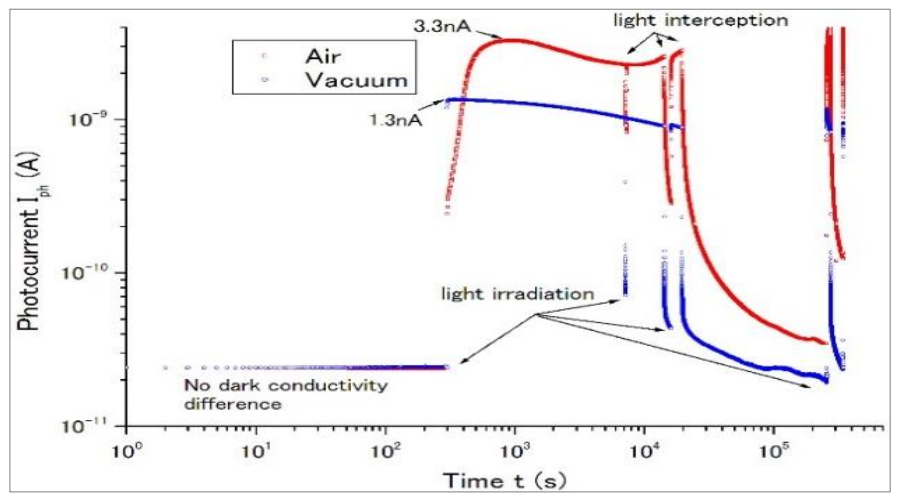

Figure 7. Photoconductivity in vacuum and air conditions

To further study the effect of air on the photoconductivity of TPD, $\mathrm{O}_{2}$ gas and $\mathrm{N}_{2}$ gas were added at $6 \mathrm{kPa}$. First, the cryostat was vacuumed. Then using the manual-3-way valve, the direction was changed from the vacuum compressor to the gas canister. A fresh sample of TPD was used per experiment under the vacuum, air, $\mathrm{O}_{2}$, and $\mathrm{N}_{2}$ conditions to clearly differentiate the effect of each condition on the sample.

Figure 8 shows that a dark current of $20 \mathrm{pA}$ to $30 \mathrm{pA}$, the photocurrent is not significantly different for all four conditions. After irradiation, the photoconductivity is higher in $\mathrm{O}_{2}$ condition, followed by that in air, in vacuum, and lastly in $\mathrm{N}_{2}$. In $\mathrm{O}_{2}$, the maximum photocurrent is $10.5 \mathrm{nA}$, whereas the maximum value in $\mathrm{N}_{2}$ is only $1.5 \mathrm{nA}$. Photoconductivity degradation occurs in all conditions; however, the highest speed to degradation is observed in $\mathrm{N}_{2}$, followed by air and $\mathrm{O}_{2}$. An upward trend of photocurrent is observed in air condition after the $1^{\text {st }}$ laser interception $\left(\mathrm{t}=2 \mathrm{~h}\right.$ ). Degradation trends continue in $\mathrm{O}_{2}$ and $\mathrm{N}_{2}$. When irradiation starts after $66 \mathrm{~h}$, an upward trend in photoconductivity is observed in $\mathrm{N}_{2}$ and air. The physical phenomenon for this finding is still unknown. Light-induced photoconductivity degradation occurs in all conditions.

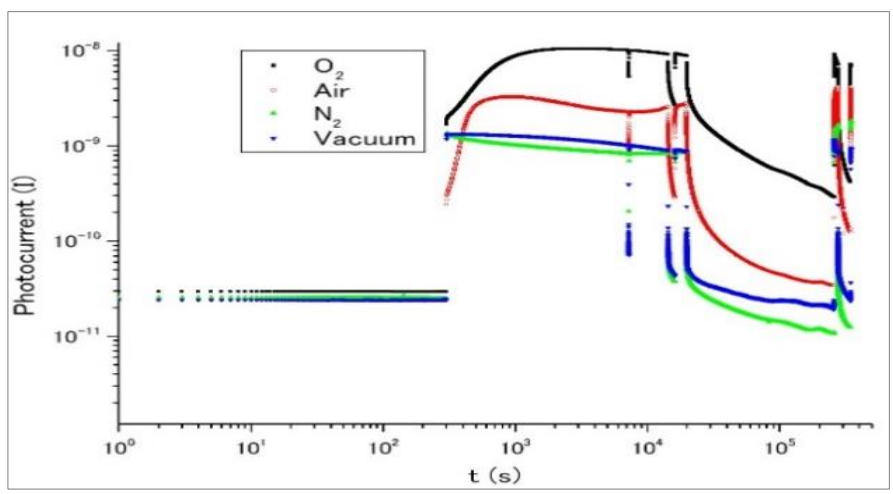

Figure 8. Photoconductivity in air, $\mathrm{O}_{2}$ and $\mathrm{N}_{2}$ conditions

$\mathrm{N}_{2}$ gas is an inactive gas that is widely used in the semiconductor industry. The amount of water vapor in $\mathrm{N}_{2}$ used in this study is 10 parts per million (ppm). The $\mathrm{N}_{2}$ gas used in this study contained only $\sim 1$ $\times 10^{13}$ water vapor molecules. In this study, $6 \mathrm{kPa} \mathrm{N} 2$ is used. Hence, the effect is actually smaller. The number of carriers is $10^{16}$. This value is still high compared with $10^{13}$, which is the same as the number of the water vapor molecules. Therefore, the effect of water vapor is insignificant in this study. 
Photoconductivity degradation is further studied because light-induced photoconductivity is a crucial characteristic of semiconductor materials. In the enlarged view of Figure 9, it shows that in almost all conditions, degradation does not recover when the laser is cut for $60 \mathrm{~s}$ after irradiation for $1 \mathrm{~h}$ and $55 \mathrm{~min}$. Thus, photoconductivity decreases when light is continuously irradiated to the semiconducting photoconductive materials. In the semiconductor industry, materials are only used after degradation is completed. However, the steady-state degradation of photoconductivity is poorly understood. Therefore, the time required for the material to recover to its original initial value after the light is cut off should be determined.

In the enlarged view of Figure 10, it is clearly seen that the light is cut off for $30 \mathrm{~min}$ after $3 \mathrm{~h}$ and $54 \mathrm{~min}$ of irradiation. Photoconductivity continues to exhibit a similar degradation pattern. Therefore, the recovery of photoconductivity to its original value in TPD requires longer than $30 \mathrm{~min}$. To further investigate the permanence of photoconductivity degradation, the light is again cut off for $66 \mathrm{~h}$ after $4 \mathrm{hr}$ and 54 min of irradiation. Figure 11 shows that most of the conditions recover when the photocurrent is higher compared with that exactly before the cut-off. The photocurrent shows an upward trend in $\mathrm{N}_{2}$. Another degradation trend is observed in $\mathrm{O}_{2}$ and vacuum conditions. The upward trend of the photocurrent is also observed in air because $78 \%$ of the air is $\mathrm{N}_{2}$. The reason for this upward trend in $\mathrm{N}_{2}$ is unknown. A graph is plotted to show the degradation phenomenon in all conditions. The data from the maximum photocurrent point until the cut off are fitted to a stretch function. Only the attenuation effective response time $\mathrm{T}$ and the dispersion parameter $\beta$ are measured in this experiment. The $\mathrm{T}$ and beta for both photoconductivity degradation (Table 2) and residual decay (Table 3) are obtained by fitting the graph with the stretched exponential function as shown in Figure 12 to measure attenuation response time and dispersion time. The results from the fitting are shown in Table 3.

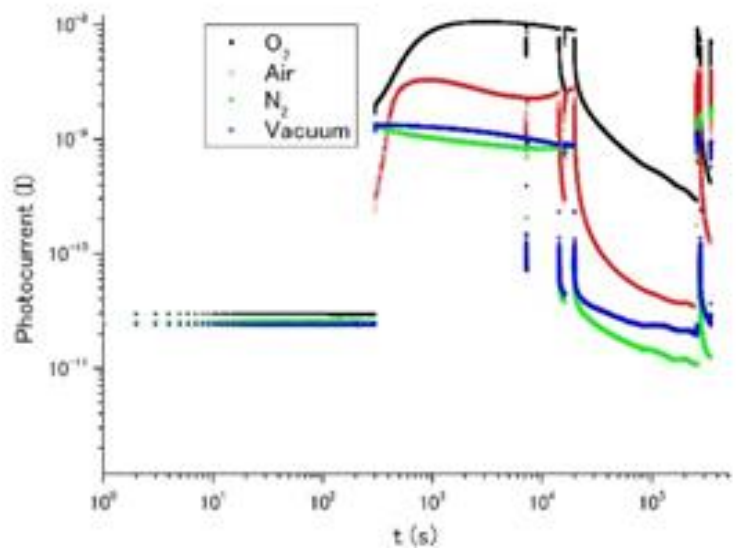

Figure 9. Photoconductivity degradation after $60 \mathrm{~s}$ light interception

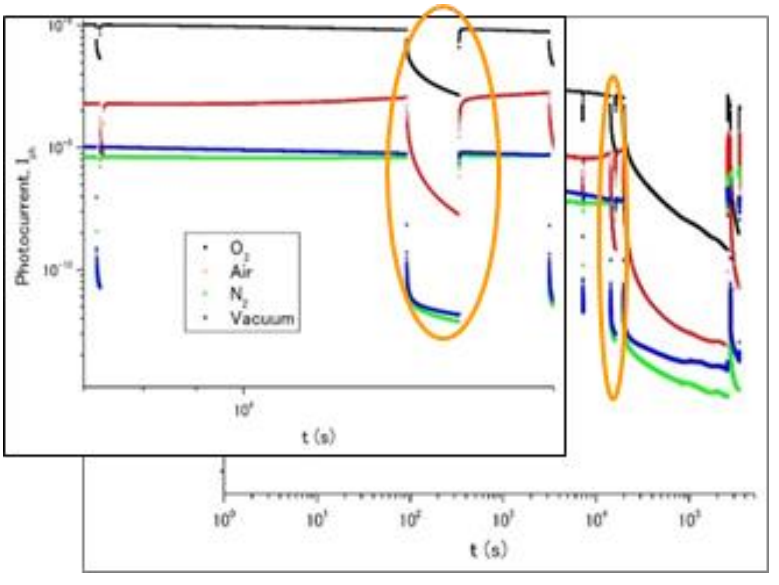

Figure 10. Photoconductivity degradation after 30 mins light interception

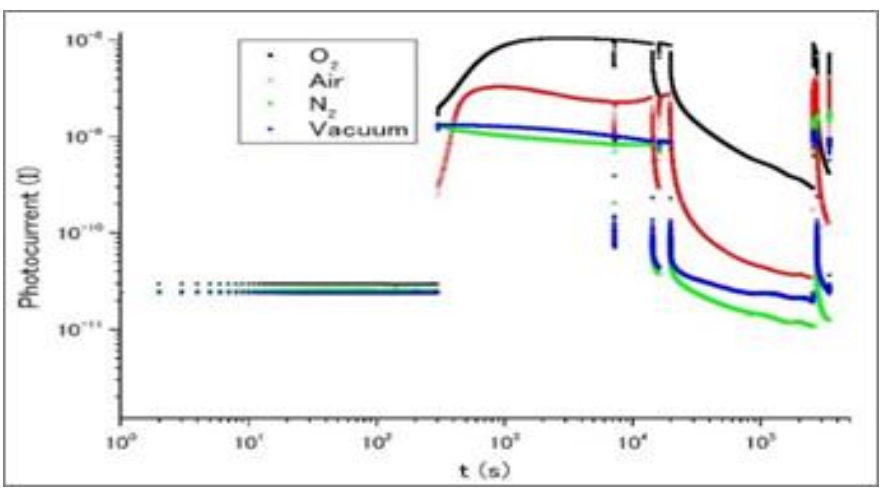

Table 2. ${ }_{\mathrm{T}}$ and $\beta$ Value for Photoconductivity Degradation

\begin{tabular}{lcc}
\hline \multicolumn{3}{c}{$\mathrm{f}_{2}(\mathrm{t})=\mathrm{A} \exp \left[-(\mathrm{t} / \mathrm{T})^{\beta}\right]$} \\
\hline Condition & $\mathrm{T}[\mathrm{s}]$ & $\beta$ \\
\hline Vacuum & 8138 & 0.80 \\
Air & 2039 & 1.28 \\
$\mathrm{O}_{2}$ & 12476 & 1.49 \\
$\mathrm{~N}_{2}$ & 724 & 0.47 \\
\hline
\end{tabular}

Figure 11. Photoconductivity degradation after 66 hours light interception 


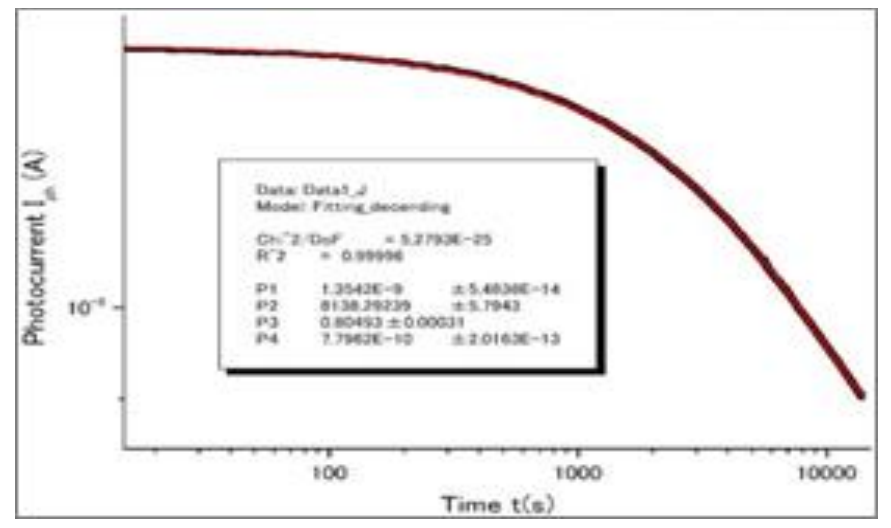

Table 3: $\mathrm{T}$ and $\beta$ Value for Residual Decay in TPD

\begin{tabular}{lcc}
\hline \multicolumn{3}{c}{$f_{2}(t)=A \exp \left[-(t / T)^{\beta}\right]$} \\
\hline Condition & $\mathrm{T}[\mathrm{s}]$ & $\beta$ \\
\hline Vacuum & 0.07 & 0.1 \\
\multirow{2}{*}{ Air } & & 0 \\
& 147 & 0.2 \\
$\mathrm{O}_{2}$ & & 9 \\
& 2139 & 0.3 \\
$\mathrm{~N}_{2}$ & & 9 \\
& 478 & 0.2 \\
& & 2 \\
\hline
\end{tabular}

Figure 12. Fittings by origin lab

\section{CONCLUSION}

This paper present photoconductivity measurement of TPD under vacuum, air, nitrogen and oxygen conditions. First, air produces about 1.5 times higher photocurrent compare to in vacuum condition. Second, conductivity in dark shows no difference in all conditions. Third, light induced photoconductivity occurs in all conditions. Then, $\mathrm{O}_{2}$ increases the photoconductivity and give a larger ${ }_{\mathrm{T}}$ in both degradation and residual decay. On top of that, $\mathrm{N}_{2}$ and $\mathrm{O}_{2}$ give longer effective response time during residual decay of photoconductivity. Besides, degradation occurs in all conditions and its recovery takes more than 65 hours.

The increase in photoconductivity in air is reversible, indicating that this response is a physical reaction. Considering that oxygen attracts electrons, the number of photo-created electrons decreases with $\mathrm{O}_{2}$ content and increases the lifetime of holes, which are the dominant carriers in TPD films.

\section{REFERENCES}

[1] Hiroo, I. Laureate Lectures, New Developments in Organic Semiconductor and Conductors, Kyoto Prize Workshop in Advanced Technology. 2007.

[2] Mohan, V. J. Organic semiconductors: past, present and future. Electronics. 2014; 3: 594-597.

[3] Stephen, R. F. The path to ubiquitous and low-cost organic electronic appliances on plastic. Nature, 2004; 428, 911-918.

[4] Day, J., Subramaniam, S. Anthony, J. E., Lu, Z., Twieg, R. J. \& Ostroverkhova, O. Photoconductivity in organic thin films: from picoseconds to seconds after excitation. Journal of Applied Physics. 2008: 103.

[5] Rosalba, L. A study on defects in organic semiconductors for field effect transistors. Thesis of Doctorate. 2013.

[6] Sato, T., Yonezawa, K., Shimakawa, K \& Naito, H. Photoconductivity in organic TPD film: effect of photo adsorption of $\mathrm{O}_{2}$ and $\mathrm{N}_{2}$. Journal of Non-Crystalline Solids, 2008; 2866-2869.

[7] Irkhin, P., Najavoc, H \& Podzorov, V. Steady-state photoconductivity and multiparticle interactions in highmobility organic semiconductors, Sci Rep. 2015: 5.

[8] Rudati, P. S., Mueller, D. C. \& Meerholz, K. The I-V characteristics of organic hole-only devices based on crosslinked hole-transport layer. Journal of Applied Research and Technology, 2015; 13(1), 253-260.

[9] Shimakawa, K., Nayan, N. A., Yonezawa, K \& Sato, T. Dynamics of photoconductivity in organic TPD films. Journal of Optoelectronics and Advanced Materials 2007; 9(1), 65-69.

[10] June, R.K, Cunningham, J.P, Fyhrie, D.P, Novel Method for Curve fitting the Stretched Exponential Function to Experimental Data. Biomed Eng Res. 2013 December 1; 2(4): 153-158. 